\title{
Modal Auxiliaries as Stance-Taking Devices in Linguistics Re- search Articles: A Functional Contrastive Analysis
}

\author{
Fatemeh Moafian, Amrollah Talati-Baghsiahi and Nahid Yarahmadzehi (Chabahar)
}

\begin{abstract}
The current paper is to report a study on modal auxiliaries as stance-taking devices applied in English linguistics research articles by native and Persian non-native writers. In doing so, 20 (10 in each group) research articles were selected. The corpora were detected for the occurrences of modal auxiliaries both mechanically and functionally. The obtained data was analyzed and the frequency and proportionate distribution of the individual modals with respect to each other were determined. The results uncovered some similarities and differences between the two groups of writers. Both groups of writers used modal verbs in the epistemic functions more than their deontic ones. Moreover, they preferred hedging strategies to boosting ones. Native writers, however, used modal verbs far more frequently than nonnative ones. The proportion of modal verbs did not follow a similar pattern in the two groups of texts. The findings offered some implications for English language education system contributors including material developers, lecturers, teachers and researchers. The interpretations and implications of the study were discussed with respect to the obtained results.
\end{abstract}

\section{$1 \quad$ Introduction}

Generally, academic written discourse is an interpersonal activity like any other verbal interactions. It is a phenomenon which develops interactively between the participants, with the writers expressing not only factual information through propositions but also information including their feelings, attitudes, evaluation and commitments regarding what is being communicated and towards the audience. The former information is mostly created according to the ideas and thoughts to be transacted and the latter information formulated the interlocutors' stances towards both those ideas and the readers, which are stated and realized linguistically through various lexico-grammatical resources in the texts (Almeida/Vazquez 2010). Stance which is realized through linguistic properties to signal authors' attitude and reaction in texts have drawn growing attention in the literature for a long time (Ahmad/Mehrjooseresht 2012). Investigating stance includes the study of linguistic alternatives in discourse, which may denote meanings other than literal (Almeida/Vazquez 2010). Not only research authors are required to express the knowledge facts in their study, but also they have to take stances towards earlier findings stated by others as well as indicate their attitudes towards their own knowledge claims (Ahmad/Mehrjooseresht 2012). Among the common resources the writers employ to signal their stances towards different propositions are modal verbs. 
One of the specific features of modal verbs is that they always occur with the main verb in which the proposition of the sentence is included. So, in every sentence, it is easy and possible for academic writers to show their stances towards propositions via using modal verbs. Orta (2010: 79) maintained "without a modal verb, most verb phrases include only a marking of time orientation and not an overt expression of stance". Furthermore, modal auxiliaries as stance devices are typical in academic texts. However, they are ambiguous in their meaning as they are multi-functional and polysemous (Chen 2012; Hyland/Milton 1997; Lorenzo 2008); this in turn leads to great difficulties for the scientific authors, specifically the EFL ones, in negotiating the meaning with the readership. This shows the significance of working on EFL academic discourses among which are Persian writers'. The reason why Persian writers' texts have been selected to be compared with native English ones is the fact that, in Persian, the number and the variety of modal verbs are not as many as those in English. Additionally, some modal verbs in English do not have any equivalents in Persian. Accordingly, mastering proper employment of English modal verbs in academic writing seems to be an area of difficulty for Persian writers. Thus, this area deserves more investigations. With this in mind, the current study, using a contrastive linguistics analysis, aims at investigating the deployment of the modal auxiliaries as one type of stance-taking resources in English linguistics articles written by native and nonnative (Persian) authors. Moreover, they are examined from the perspective of hedging and boosting strategies.

\section{Literature review}

\subsection{Modality and modal verbs}

"Modality" denotes the meaning which is expressed by linguistic sources that signal individual stance. Its realization in the text permits propositions to be identified as assertions or rejections of "possibility, impossibility, contingency, or necessity other than simple declarations of the fact" (Hsieh 2009: 1).

Authors exploit modality expressions to transfer their personal beliefs, feelings and judgments concerning specific propositions. Two prominent pragmatic functions are accomplished by modal expressions through the text. The writers can use them: 1) to assess their knowledge claims and make clear the degree of their confidence in those assertions and 2) to express respect and humility towards their readers. That is, modal expressions aid authors to communicate their stances towards both their propositions and their addressees. These two applications establish a rhetorical characteristic that can have a substantial impact on a reader's appraisal of discourse (Lorenzo 2008).

One channel of expressing modality is modal auxiliary verbs. Modal auxiliaries are believed to be polysemous. That is, they can be applied in a wide range of meanings such as obligation, necessity, permission and ability (Orta 2010). Based on their meaning, three major classes are considered for modal verbs in the Longman Grammar of Spoken and Written English as follows: First, permission, ability and possibility are expressed by can, could, may and might; second, obligation and necessity are conveyed by must and should; and third, volition and prediction are articulated by will, would, and shall (Biber et al. 1999). 
The authors can assess a certain situation with respect to possibility, probability, permission, volition, obligation and necessity, using modal auxiliary devices. To put it in different words, all the notions mentioned above carry the writers' subjective attitudes towards their statement, representing the authors' personal beliefs and their relationship with reality (Orta 2010).

\subsection{Stance and modal verbs}

In any situation of interaction in the mode of writing or speaking, not only information is contained in the linguistic message, but also authors' relationship with that information as well as with their addressees is included in it whether implicitly or explicitly. Based on the cultural values and conventions, the format of certain types of texts or particular discourse genres are also shaped; for instance, giving a person an address includes specific linguistic principles which vary considerably from those for presenting a sermon or giving a political speech. Both the personal and cultural conventions consistently influence the linguistic form and content of the text, which in turn they are broadly referred to as the speaker or the writer's stance (Reilly/Zamora/McGivern 2005). In fact, stance refers to "the level of personal involvement of the writer or speaker with the text, as well as his or her moral evaluation, degree of certainty, and/or emotional perspective and response to the content of the text" (Reilly/Zamora/McGivern 2005: 186).

Hyland (1999) categorizes stance into three components: evidentiality, affect, and relation. By "evidentiality", he means the author's declared commitment to the truth of propositions and their planned manipulation for interpersonal aims. "Affect" refers to the expression of a variety of attitudes, involving beliefs, emotions and viewpoints. "Relation" contains the degree of detachment or intimacy between the writer and the addressee and, in general, the distance which writers keep with readers.

Stance can be linguistically realized through various forms including lexical markers, grammatical markers, and person markers. Lexical stance is manifested via verbs, nouns and adjectives as the evaluative lexical elements. Grammatical stance is realized via the following categories: “(a) stance adverbials, (b) stance complement clauses, (c) modals and semimodals, (d) stance noun + propositional phrase, and (e) premodifying stance adverb (stance adverb + adjective or noun phrase)" (Almeida/Vazquez 2010: 1173).

The focus of the current study is modal verbs which is a subcategory of grammatical stance devices. Two different kinds of meanings can be basically expressed by modal verbs, which are referred to as deontic and epistemic. The former represents a degree of desirability via obligation, volition and permission. The latter, conveying the degree of probability includes beliefs, necessity, the logical possibility, predictability and hypothetical meaning (Orta 2010). This division is in line with Biber et al.'s intrinsic and extrinsic modality:

Each modal can have two different types of meaning, which can be labeled intrinsic and extrinsic (also referred to as 'deontic' and 'epistemic' meanings). Intrinsic modality refers to actions and events that humans (or other agents) directly control: meanings relating to permission, obligation, and volition (or intention). Extrinsic modality refers to the logical status of events or states, usually relating to assessments of likelihood: possibility, necessity, or prediction.

(1999: 485) 
Modal verbs in different categories and meninges, as mentioned above, are summarized in Table 1:

\begin{tabular}{lll}
\hline Modal verbs & Deontic (intrinsic) meaning & Epistemic (extrinsic) meaning \\
\hline can, could, may, might & permission, ability & possibility \\
must, should & obligation & necessity \\
will, would, shall & volition & prediction \\
\hline
\end{tabular}

Table 1: Modal verb and their different types of categories and meanings

As Table 1 shows, modal verbs are employed to convey a writer's stance; they denote either deontic modality including meanings such as necessity, permission and obligation, or epistemic modality including the degree of certainty of the knowledge fact. Most verb phrases without a modal verb do not carry an overt expression of stance but a marking of time orientation (Orta 2010).

In most cases, the linguistic structure representing the proposition follows the stance marker. In a similar vein, the main lexical verb follows modal verbs and thus modal verbs typically precede the assertion of new information in the clause. This arrangement of components of the clause demonstrates the crucial function of stance expressions as a framework for the perception of the propositional content. To put it another way, authors first specify their personal stance, through which they encourage the audiences to interpret the following proposition from a similar point of view (Orta 2010).

\subsection{Hedging and boosting and modal verbs}

In written discourse, "hedges" are usually defined as various syntactic and lexical structures applied to moderate writers' assertions or statements and to reduce their responsibility for the truth value of the claims by other scholars (Hinkel 1997, 2005). On the other hand, "boosters" denote certainty and highlight the force of propositions (Hyland/Tse 2004). Hedging and boosting are employed as communicative techniques to increase or decrease the force of propositions. Due to their contribution to proper rhetorical and communicating tenor which expresses both affective and epistemic meanings, they are prominent in academic texts. In other words, they express both the author's level of assurance in the accuracy of a proposition and their attitude to the reader (Hyland 1998a).

Boosters can be utilized to create trust and certainty in readers and establish an impression of confidence, assurance and conviction (Hyland 1998b). Hyland points out that boosting devices help to reinforce statements and demonstrate the authors' commitment to their propositions. He maintains although such expression of the writer's belief seems to leave not much room for the interpretations of the addressee, boosters can be applied as a tool to engage the authors with their audience and establish interpersonal solidarity (Hyland 1998a).

In academic writing, the authors need to try to persuade their readers of the truth-value of their assertions. This is especially true when the writers are presenting the statements containing new knowledge. The major functions of boosters will concern encouraging and satisfying the addressees of the content which is supported by the information in the text (Vázquez/Giner 2009). On the other hand, hedging is the representation of authors' tentative- 
ness, and it is one of the main features of academic writing in which assertions are seldom made with objective appraisal of their reliability (Hyland 2006).

Many knowledge claims in the world of science do not survive over a long period of time. They are substituted by those assertions which enjoy a greater explanatory efficiency, and it is always possible that scientific statements will challenge or oppose the opinions of one's colleagues. The fact that readers have the capability to deny the author's assertions indicates that they play a dynamic and constitutive part in the way the writers formulate them. Due to the writer's expectation of this potential contradiction on the part of the reader, hedging has become focal to academic writing. So, hedging devices aid authors not to take the whole personal responsibility for the claims so as to save their face and confine the negative consequences that may arise from categorical commitments. Ultimately, hedging can be considered "reader-oriented" since it helps to improve a writer-reader relationship by making the writer attend to the readers' need for deference and cooperation in confirmation of his statements (Hyland 2006).

Moreover, hedging is one of the many strategies of politeness which writers apply to mitigate the threat that academic claims exert to the "face" of readers, hence, avoiding face-threatening acts throughout the text (Brown/Levinson 1987). In science, hedging plays an important role in readers' acceptance of statements expressed by academic writers via permitting authors to represent their claims with proper precision, tentativeness and modesty (Hyland 1996).

There are different ways that boosting and hedging can be realized linguistically in discourse, including lexical and grammatical resources such as modal adverbs (e. g. perhaps, probably), modal adjectives (e. g. possible, probable), modal auxiliaries, etc. (Hu/Cao 2011). Among the mentioned lexical and grammatical resources, the current study chooses to investigate modal auxiliary verbs as hedging and boosting devices expressing the authors' stances in scientific discourse. The modal verbs including can, could, may, might, would and should are categorized as hedging devices and the modal verbs involving will, must and shall are considered as boosting resources (Lorenzo 2008).

\subsection{Previous studies}

Stance-taking has been scrutinized from different perspectives over the last several years in literature. For instance, Ahmad and Mehrjooseresht (2012) examined engineering doctoral thesis abstracts to understand the way L2 writers take their stance via adverbials through the texts. They found that the writers employ adverbials with a high frequency to show their degree of certainty about the propositions. Almeida and Vazquez (2010), in a study on modal verbs as stance markers, documented that the speakers' subjectivity is realized through modal verbs. Huschová (2015) investigated 400 contextualized instances of can/could and may/might extracted from linguistic academic books in terms of the function of possibility. She attempted to investigate the relationship between the contextual factors and semantic interpretation of the given modal verbs. The results indicated that generally the modal verbs were used denoting predominantly root (or non-epistemic) possibility in the texts. The findings also revealed that unlike may/might which appeared to mark both epistemic and root possibility, can/could conveyed no epistemic possibility. She also concluded that the four modal 
verbs appeared to be closely associated with the notion of hedging. In other words, they were mostly used to show the writer's detachment from the propositions.

There are also some studies investigating the differences in the employment of stance-taking makers from a cross-cultural outlook. For instance, in a contrastive analysis study, Chen (2012) examined epistemic stance markers in English argumentative texts written by native speakers and Chinese non-native speakers in four proficiency levels. The findings showed a great similarity in using epistemic stances between native and non-native students' written texts. Hyland and Milton (1997) also analyzed the epistemic stance markers in the argumentative texts written by English native and Chinese non-native speakers. In contrast to Chen's findings, the results revealed that the non-native writers employed a limited number of epistemic stance markers, consequently, making stronger claims in their writing. In a similar study, Orta (2010) reported on the quantitative and qualitative analysis of modal auxiliaries as a source of epistemic stance in a corpus of English research papers written by Spanish and English writers. Orta's findings demonstrated noticeable differences between the two groups of writers in the employment of modal auxiliaries. More specifically, Spanish authors indicated a different strategy in the employment of hedges and boosters. Hence, they had problems in establishing an appropriate tenor when they wrote in English.

As the review of the related literature reveals, although the studies on stance markers are not limited, they differ with respect to the methodologies and the aims they followed. Three of the most noticeable differences among these studies are genre types, the types and number of stance markers, and culture on which they focused. The different discourse types which were investigated include doctoral thesis abstracts (Ahmad/Mehrjooseresht 2012), English recipes (Almeida/Vazquez 2010), expository (Hyland/Milton 1997) and argumentative discourse (Chen 2012; Hyland/Milton 1997), Business Management research articles (Orta 2010), and linguistics books (Huschová 2015). Regarding the types and number of stance markers, Chen (2012) and Hyland and Milton (1997) investigated the five categories of stance markers including modal verbs, adverbials, lexical verbs, adjectives, and nouns; Almeida and Vazquez (2010), Orta (2010), and Huschová (2015) studied modal verbs, however, Huschová investigated only may/might and can/could of the modals; Ahmad and Mehrjooseresht (2012) examined adverbials. Some limited their studies to one culture (Ahmad/Mehrjooseresht 2012; Almeida/Vazquez 2010; Huschová 2015), whereas the others carried out their research interculturally (Chen 2012; Hyland/Milton 1997; Orta 2010). Even though the reviewed studies dealt with the issue of stance-taking from different perspectives, most of them acknowledged the significance of stance-taking in various texts and discourses. On the other hand, the increasing bulk of scientific research paper publication worldwide, particularly on the part of EFL writers, signals the significance of investigating stance markers in research articles. That is because taking appropriate stances towards claims and propositions with regard to available evidence and assumptions is an area of difficulty for both native and non-native academic writers. Moreover, any erroneous stance-taking can affect the text coherence, the impact of the argument, and the judgment about writers' academic competence and can result in readers' misunderstanding (Hyland/Milton 1997). When it comes to EFL writers, the manipulation of degrees of probability appears to be more problematic. Values and preconceptions upon which writers from various cultures consider a feature appropriate for academic writing may differ culturally and rhetorically from those of native English academic writers. 
Such disparities may lead to violation of communicative norms on the part of non-native speakers and they would be considered as either discourteous or too uncertain, and as a result unconfident or naive since their writing appear as too direct (Hyland/Milton 1997). In spite of such cross-cultural realities, there seems to be a dearth of sufficient studies on the research articles inter-culturally. Orta (2010) is among those that examined this issue between Spanish and English writers and the findings revealed that there was a considerable difference between the two groups of writers in terms of stance-taking in the research articles. This shows the necessity for conducting similar research on other cultures. To fill the perceived gap, the current study seeks to investigate stance-taking in English linguistics research articles written by English and Persian scholars since, to the best knowledge of the researchers, no study has been conducted in this scope.

\section{The framework of the study and research questions}

The framework of the study has been inspired by the one used by Orta (2010). Following Orta, the present research has analyzed the data, using bottom-up and top-down strategies. That is, in the bottom-up approach, first the corpus has been analyzed and accordingly different discourse unit types have been specified. In the top-down approach, identifying the discourse unit types has preceded the analysis of the corpus. To fulfill the aims of the study the following research questions were raised:

1. What are the frequency and distribution of modal auxiliary verbs in English linguistics research articles written by native and non-native authors?

2. How do the two different groups of writers make use of modal auxiliaries regarding their epistemic and deontic meanings in English linguistics research articles?

3. What are the frequency and distribution of modal auxiliaries in terms of hedging and boosting in English linguistics research articles?

\section{$4 \quad$ Method}

\subsection{Material}

The corpus was composed of 20 linguistics research articles written in English by native and non-native Iranian writers. The articles were tried to be selected from the most widely-read and prestigious academic journals which was obtained through their impact factor, indexing and their reputation. The corpus included ten articles written by native researchers and ten articles written by Iranian authors (see Table 1). To be representative of native and non-native authors, the writers of the papers were needed to have Angelo-American and Iranian last names and to be affiliated with the institutions in countries where these languages are spoken as the first language. Additionally, the attempt was made not to choose more than one article by the same author. All the papers were published between 2009 and 2014.

Excluding abstracts, notes, bibliographies, quotations, tables and figures, the study took only the body of the research articles into consideration. Because of the unequal sizes of the papers (the number of words) in the two corpora, the relative frequency of devices rather than the total number of occurrences was put into analysis. 


\begin{tabular}{|l|c|l|c|}
\hline Corpus & words & \multicolumn{1}{|c|}{ sources/journals } & no. of articles \\
\hline ET & & Language Sciences & 6 \\
& & Language \& Communication & 1 \\
& & Journal of Pragmatics & 2 \\
Total & \multirow{2}{*}{89196} & Lingua & 1 \\
\hline ET (Iranian) & & Language Sciences & 10 \\
& & International Journal of Lexicography & 2 \\
& & Iranian Journal of Applied Language & 6 \\
Total & & Studies & 1 \\
\hline Totals & 141767 & Studia Linguistica & 10 \\
\hline
\end{tabular}

Table 2: The sources of the two corpora

\subsection{Procedure and data analysis}

All occurrences of modal auxiliaries including can, could, may, might, shall, should, will, would, and must were counted in the two corpora. This has been done without considering their functions in the texts to find out merely the general pattern and distribution of modal auxiliaries in the two text groups. Counting and recording the total number of the frequencies of auxiliary verbs in the discourse, the authors calculated their relative frequency per one thousand words due to the inevitably unequal sizes of the samples (NE $=89196$ words, NNE $=52571$ words).

In order to identify the proportion of each auxiliary in its own respective group, the percentage of every auxiliary out of the total number of auxiliaries was computed in that group. The results are presented graphically in two colored pie charts to help the readers to make a clear comparison between the two groups (see Figures 1 and 2).

In the next phase of the study, the modal auxiliaries were counted and analyzed functionally. Since modal auxiliaries are multi-functional and the different meanings assigned to each auxiliary originate from the contexts of use, a careful manual analysis was conducted concerning their functional meaning. Consequently, the occurrences of every modal auxiliary were computed based on their different functions presented in Table 1. Then, the relative frequencies of the different functions per one thousand words were calculated so that we could compare the distributions of auxiliaries in the two corpora according to their different meanings. The findings are illustrated in colored bar graphs to ease comparison (see Figures 3, 4, 5, 6, 7 and 8).

In the last stage of the study, modal auxiliaries were investigated according to their hedging and boosting functions to identify the writers' stances taking procedures towards their propositions. In doing so, the relative frequency of hedging and boosting resources per one thousand words and their percentage in their own group were estimated. This process was carried out both for the total number of hedges and boosters and for each modal auxiliary with the functions of hedging and boosting. The results are represented in Tables 3, 4 and 5. 


\section{$5 \quad$ Results}

In native English (NE) texts, modal auxiliaries occurred in a frequency of 13.05 per one thousand words while, in non-native English (NNE) texts, their frequency of occurrence equaled to 5.51 verbs per one thousand words. The general patterns of modal distributions in the two groups of texts are illustrated in percentages in Figures 1 and 2.

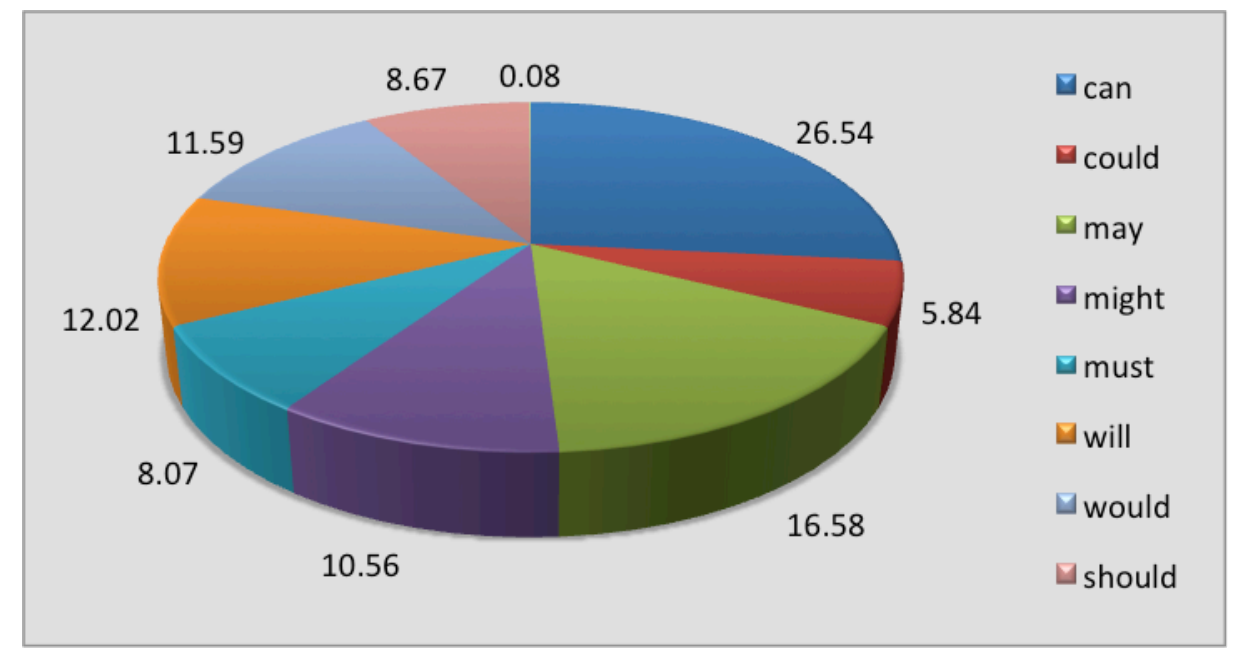

Figure 1: Percentage of modal verbs in NE texts

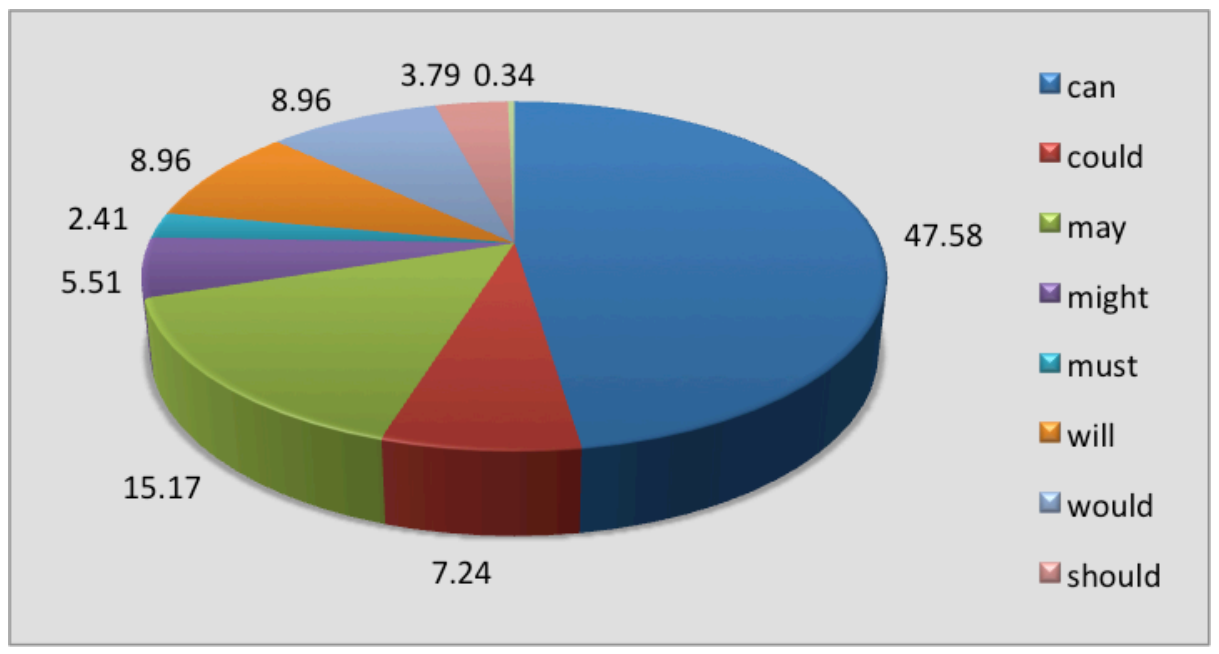

Figure 2: Percentage of modal verbs in NNE texts

As the pie chart in Figure 1 demonstrates, the modals can (26.54\%) and may (16.58\%) were more common in comparison to the other modal verbs in NE texts. At the other extreme, the modal shall $(.08 \%)$ was very scarce. The remaining modal verbs including will $(12.02 \%)$, would $(11.59 \%)$, might (10.57\%), should (8.67\%), must (8.07\%), and could (5.84 \%) were located in the middle. Concentrating on the pairs of central models, we observed that could, might, and would were less common than their counterparts. However, this was not true for shall/should $($ can $=26.54 \%$, could $=5.84 \%$, may $=16.58 \%$, might $=10.56 \%$, will $=$ $12.02 \%$, would $=11.59 \%$, shall $=.08 \%$, should $=8.67 \%$ ).

Regarding the NNE texts, the pie chart in Figure 2 illustrates that can (47.58\%) was extremely common in NNE corpora followed by may (15.17\%). At the other extreme, the modal shall $(.34 \%)$ was very infrequent. The rest of the modal verbs including will (8.96\%), would $(8.96 \%)$, could $(7.24 \%)$, might $(5.51 \%)$, should $(3.79 \%)$, and must $(2.41 \%)$ were placed 
somewhere in between. With respect to the pairs of central models, a different pattern was observed, that is, for can $(47.58 \%)$ /could $(7.2 \%)$, and may $(15.17 \%)$ /might $(5.51 \%)$, the tentative/past time members were less common than their partners; for will $(8.96 \%)$ wwould $(8.96 \%)$, the so called tentative member was as frequent as its partner; for shall $(.34 \%)$ should $(3.79 \%)$, the tentative member was more frequent than its counterpart.

Comparing the results of the two corpora, we see that although can was the most frequent in the two types of texts, its proportion in NNE texts was approximately twice as much as its proportion in NE texts. The second most frequent modal verb, may, was employed in the two types of texts with nearly the similar proportions. The next two more common modal verbs in NE and NNE texts were will and would. Similarly, the authors of the two types of texts made use of them with not much different proportions $\left(\right.$ will $_{\mathrm{NE}}=12.02 \%$ vs. will $_{\mathrm{NNE}}=8.96 \%$; would $_{\mathrm{NE}}=11.59 \%$ vs. would $\left._{\mathrm{NNE}}=8.96 \%\right)$. The rank order of the next more frequent modal verbs applied in the two corpora was different. That is, in NE texts, might (10.56\%) was followed by should $(8.67 \%)$, must $(8.07 \%)$, and could $(5.84 \%)$ while, in NNE texts, could (7.24 \%) was followed by might (5.51\%), should (3.79\%), and must (2.41\%). The four mentioned cases were employed with higher proportions by NE writers than those applied by NNE authors. Untimely, shall was the last and the least frequent modal verb in the two groups; however, the proportion of this modal was less in NE texts than NNE texts. All in all, unlike Persian writers who used can and shall in higher proportions than their native English counterparts, the NE authors used the rest of modal verbs (could, may, might, must, should, will and would) in higher proportions in their writings.

The paper also investigated the overall distribution of the modal verbs according to their epistemic (extrinsic) and deontic (intrinsic) meanings. Can, could, may, and might can denote three different meanings, namely, permission, ability, and possibility. In these four modals, deontic (intrinsic) meaning entails permission and ability, and epistemic (extrinsic) meaning involves possibility (see Table 1). The following examples demonstrate the realization of different types of meanings for these modals in the corpora.

(1) Further, these contents must possess sufficient differences among their varieties such that competent speakers can distinguish them from one another (deontic: ability, NE texts).

(2) This type of compound-verb may be formed by the following syntactic patterns (epistemic: possibility, NNE texts).

(3) I told them that [...], and that they could include any amount of detail and take as much time as needed to talk about the film (deontic: permission, NE texts).

(4) Based on the findings, the linguistic nature of pragmatic strategies might be affected by the cultural schemas of a speech community (epistemic: possibility, NNE texts).

Figures 3 and 4 indicate the distribution of the four modal verbs per one thousand words based on their functional usages in the two corpora. 

as Stance-Taking Devices in Linguistics Research Articles
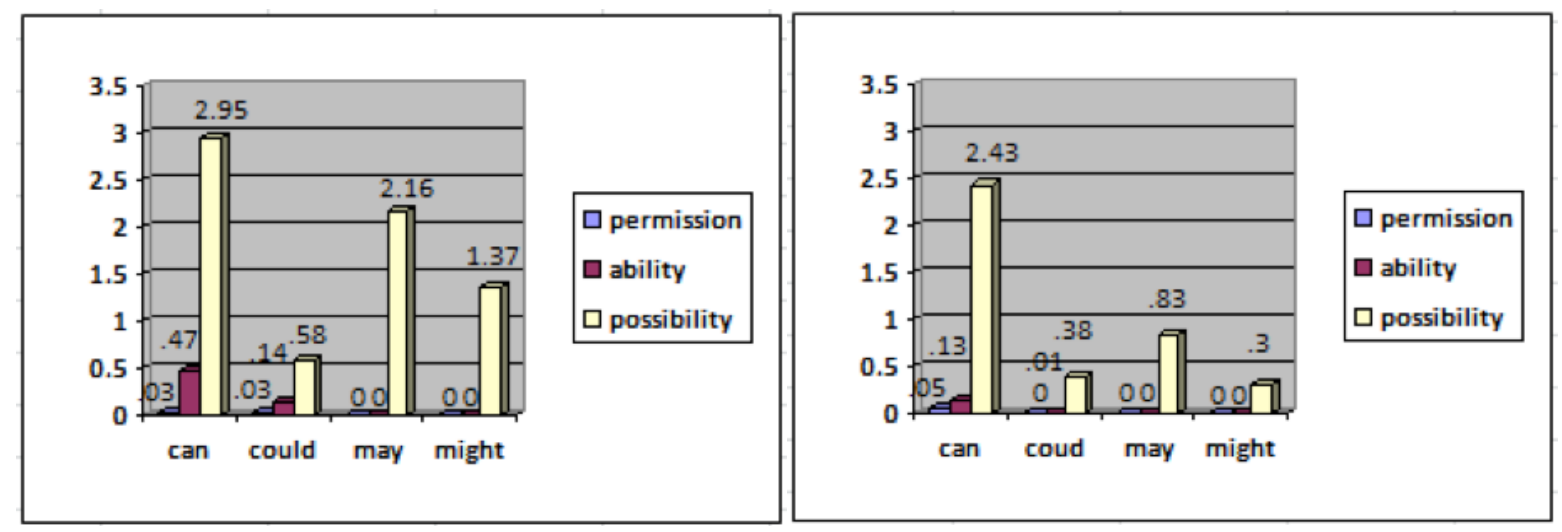

Figure 3: The functional distribution of can could, may and might in NE Texts/Figure 4: The functional distribution of can, could, may and might in NNE Texts

As Figures 3 and 4 demonstrate, all these modals were applied in their epistemic meanings (possibility) far more than their deontic (permission, ability) meanings as expected in academic discourse. In both corpora, can had the highest frequency in epistemic (extrinsic) as well as deontic (intrinsic) meanings in comparison with the three other modals. However, NE writers tended to utilize can more than their NNE counterparts in both intrinsic (.50 vs .18 per one thousand words) and extrinsic ( 2.95 vs. 2.43 per one thousand words) meanings. Although the two groups of writers used could in the same pattern, NE texts enjoyed relatively more occurrences of could in both deontic and epistemic meanings (Permission ${ }_{\mathrm{NE}}=.03$ vs. Permission ${ }_{\mathrm{NNE}}$ $=0 ;$ Ability $_{\mathrm{NE}}=.14$ vs. Ability $\mathrm{NNE}=.01$ Possibility $_{\mathrm{NE}}=.58$ vs. Possibility $\mathrm{NNE}_{\mathrm{NNE}}=.38$ per one thousand words). May and might were merely used in epistemic meaning in the two types of texts. However, the two modals were favored far more by NE authors ( $y_{\mathrm{NE}}=2.16 \mathrm{vs}$.

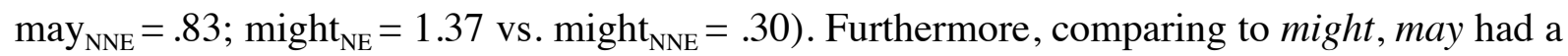
much higher frequency in both corpora.
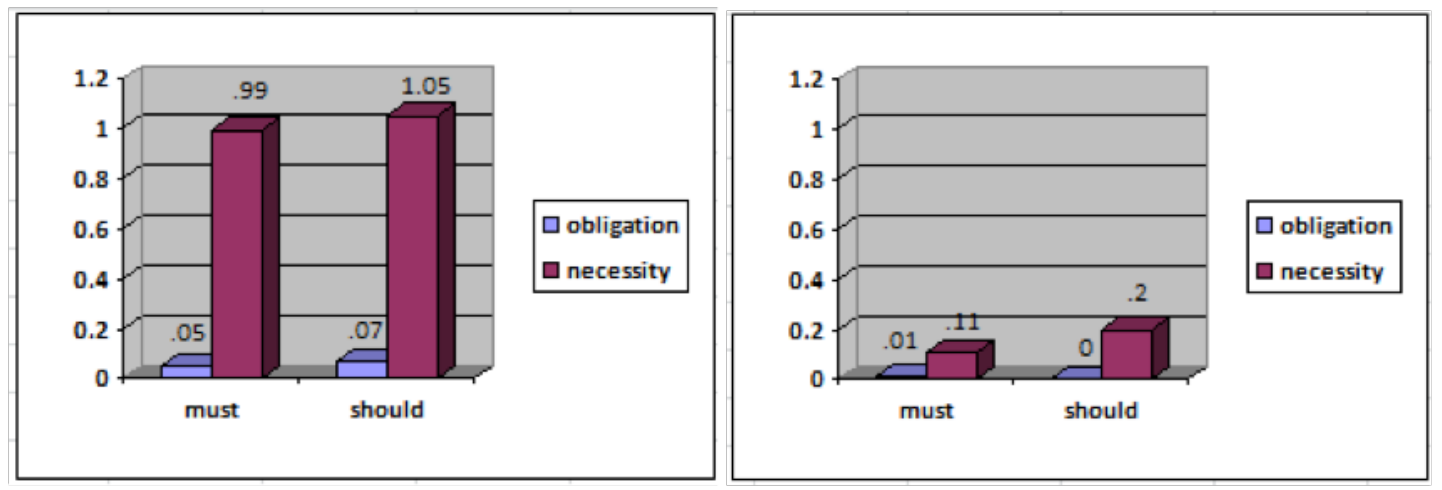

Figure 5: The functional distribution of must and should in NE texts/Figure 6: The functional distribution of must and should in NNE texts

For must and should, deontic meaning entails obligation and epistemic meaning involves necessity (see Table 1). The examples below show the realization of different types of meanings for these modals in the corpora.

(5) Students are asked (or required) to evaluate their classes and their instructors' teaching performance at the end of a term. However, they must do so anonymously via written evaluations (deontic: obligation, NE texts). 
(6) When the LVs can case mark the arguments of PVs we should expect them to be able to case mark the DP PVs too, provided that the PV is predicative (epistemic: necessity, NNE texts).

As Figures 5 and 6 show, in both texts, must and should were used in their epistemic meaning far more than their deontic meaning. In addition, both groups of writers were willing to use should more than must in the epistemic meaning (necessity). Yet, NE writers inclined to make use of must and should in both obligation and necessity functions several times as many as NNE writers did (Epistemic: must $\mathrm{NE}_{\mathrm{NE}}=.99$ vs. must $_{\mathrm{NNE}}=.11$; should $_{\mathrm{NE}}=1.05$ vs. should $\mathrm{NNE}=$ .2) $\left(\right.$ Deontic: must $_{\mathrm{NE}}=.05$ vs. must $\mathrm{NNE}=.01$; should $_{\mathrm{NE}}=.07$ vs. should $\left._{\mathrm{NNE}}=0\right)$.
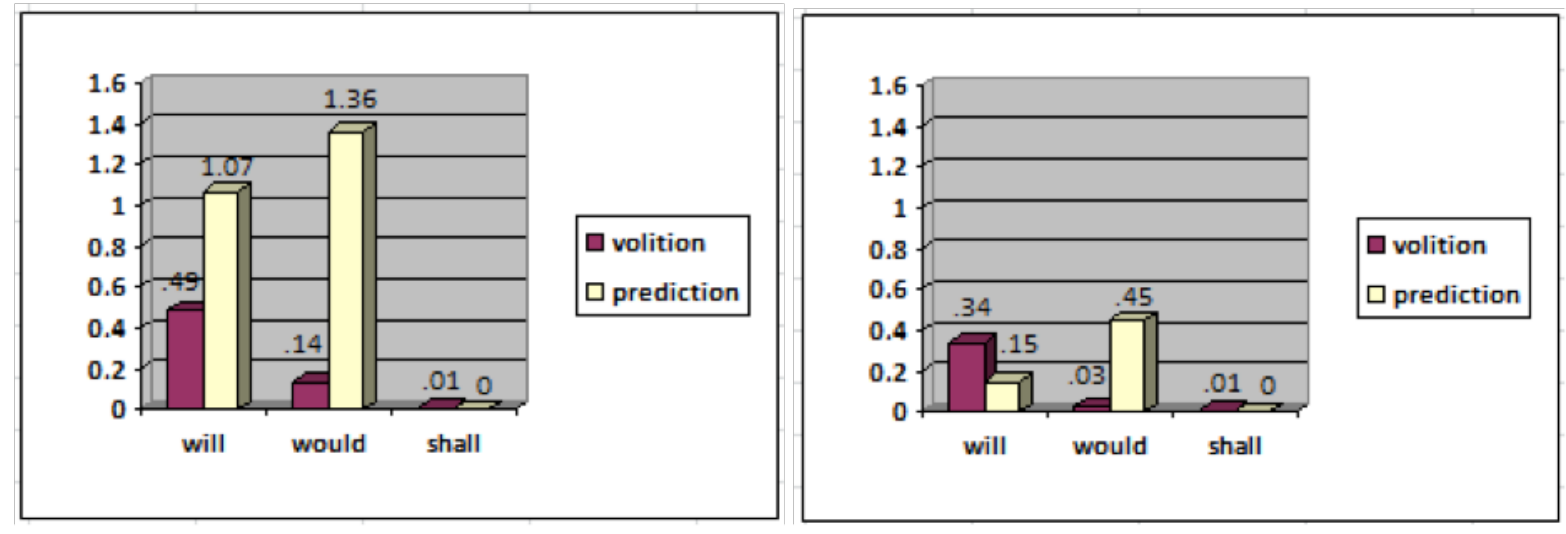

Figure 7: The functional distribution of will, would and shall in NE texts/Figure 8: The functional distribution of will, would and shall in NNE texts

Will, would, and shall denote volition as deontic and prediction as epistemic meaning (see Table 1). The following examples indicate the realization of these functions in the corpora.

(7) Prior to watching the films, the participants were told that they were about to watch a short film and that they would immediately be asked to recount the story to the researcher in as much detail as possible (deontic: volition, NNE texts).

(8) When a particular system as a whole approaches a low-dimensional description, it will typically result in many specific articulatory or auditory attractors (Epistemic: prediction, NE texts).

According to Figures 7 and 8, will (Epistemic: $\mathrm{NE}=1.07$ vs. NNE $=.15$; Deontic: $\mathrm{NE}=.49$ vs. $\mathrm{NNE}=.34$ ) and would (Epistemic: $\mathrm{NE}=1.36$ vs. $\mathrm{NNE}=.45$; Deontic: $\mathrm{NE}=.14$ vs. $\mathrm{NNE}$ $=.03$ ) were used much higher in NE texts in both deontic and epistemic meanings and shall (Epistemic: $\mathrm{NE}=.01 \mathrm{vs}$. NNE $=.01$; Deontic: $\mathrm{NE}=0$ vs. $\mathrm{NNE}=0$ ) was not favored by the two groups of writers; it had the same frequency in the two types of texts in volition function and it did not occur in either types of texts in prediction function. In NE corpora, will in its prediction meaning was applied almost twice as many as its volition meaning while, in NNE texts, it was used in its volition meaning nearly twice as many as its prediction meaning. In both groups of research articles, would was employed in its prediction function far more than its volition meaning.

In the ongoing phase of the paper, a movement was made from a merely textual analysis to a pragmatic/rhetorical one, that is, from the number of tokens of modal auxiliaries and their proportions applied by the two groups of writers to the ways the stances were taken by the 
authors towards the content and the audience. To this aim, the modal verbs were analyzed from the perspective of their epistemic meanings through their realization in the form of hedging and boosting markers in the context. That is, the modal verbs denoting deontic meanings were not counted here. Accordingly, a careful manual analysis was conducted focusing on the functional meaning of the modal verbs. The modal verbs including can, could, may, might, would, and should were considered as hedging devices and the modal verbs involving will, must, and shall were taken as boosting markers (Lorenzo 2008). For more clarification, some examples extracted from the two corpora are presented here:

(9) Moral talk can be seen as a cultural resource which at least potentially works as an attempt to address real moral difficulties (hedging, NE texts).

(10) Based on the findings, the linguistic nature of pragmatic strategies might be affected by the cultural schemas of a speech community (hedging, NNE texts).

(11) Ultimately, vague talk of "a lot" of data must be replaced with specific talk of particular quantities (boosting, NE texts).

(12) The (c) examples in (17-17ф) indicate that the heavy verb xa:ndan and the LV kardan cannot be omitted and their deletion will result in ungrammaticality, but the deletion of the future auxiliary xa:stan does not lead to ill-formed clauses (17b, 17фb) (boosting, NNE texts).

As Table 3 demonstrates, NE authors took stance towards propositions and/or readers more than twice as many as their NNE colleagues (11.58 vs. 4.88). In comparison with NNE authors, NE writers employed more hedging and boosting resources (Hedges $_{\mathrm{NE}}=9.5 \mathrm{vs}$.

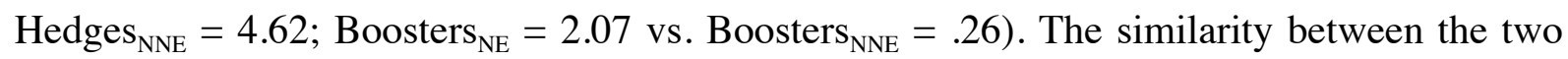
texts was that the both groups of authors used hedging markers far more than boosting markers in their writings. On the other hand, NNE authors used hedging devices in a higher proportion than that used by NE authors ( $94 \%$ vs. $82 \%$ ) while the proportion of boosting devices employed by NE writers equaled three times as much as that applied by NNE writers $(6 \%$ vs. $18 \%$ ) (see Table 3).

\begin{tabular}{|c|c|c|c|c|c|c|}
\hline & \multicolumn{3}{|c|}{ NE texts } & \multicolumn{3}{|c|}{ NNE texts } \\
\hline & Total & $\begin{array}{l}\text { Per 1,000 } \\
\text { words }\end{array}$ & Percentage & Total & $\begin{array}{l}\text { Per 1,000 } \\
\text { words }\end{array}$ & Percentage \\
\hline Hedges & 848 & 9.5 & $82 \%$ & 243 & 4.62 & $94 \%$ \\
\hline Boosters & 185 & 2.07 & $18 \%$ & 14 & .26 & $6 \%$ \\
\hline Total & 1033 & 11.58 & $100 \%$ & 257 & 4.88 & $100 \%$ \\
\hline
\end{tabular}

Table 3: The frequency and percentage of hedging and boosting modal verbs in NE and NNE texts

Table 4 indicates the proportion of hedging modal verbs in each corpus. As it is shown, can was the most favored device by both groups of writers although the Persian authors used it in a higher proportion than the other devices comparing to the native authors $(52.67 \%$ vs. $31.13 \%)$. In a similar vein, both groups applied may as the second most frequent marker though the NE writers employed it in a higher proportion than the Persian writers $(22.75 \%$ vs. $18.1 \%)$. The other hedging modal verbs were not used with the same order and proportions in the two corpora (might: $14.5 \%$ vs. $6.58 \%$; would: $14.38 \%$ vs. $9.87 \%$; should: $11.08 \%$ vs. $4.52 \%$; could: $6.13 \%$ vs. $8.23 \%$ ) (see Table 4 ). 


\begin{tabular}{llllll}
\hline & \multicolumn{2}{c}{ NE texts } & & \multicolumn{2}{c}{ NNE texts } \\
\cline { 2 - 3 } & Total & Percentage & & Total & Percentage \\
\hline Can & 264 & $31.13 \%$ & & 128 & $52.67 \%$ \\
May & 193 & $22.75 \%$ & & 44 & $18.1 \%$ \\
Might & 123 & $14.5 \%$ & 16 & $6.58 \%$ \\
Would & 122 & $14.38 \%$ & & 24 & $9.87 \%$ \\
Should & 94 & $11.08 \%$ & & 11 & $4.52 \%$ \\
Could & 52 & $6.13 \%$ & 20 & $8.23 \%$ \\
Total & 848 & $100 \%$ & 243 & $100 \%$ \\
\hline
\end{tabular}

Table 4: The frequency and distribution of the types of hedging modal verbs in NE and NNE texts

Table 5 summarizes the proportion of boosting modal verbs in each corpus. Both groups of writers used the first two boosting devices (will and must) with the same order but with a different proportion. Shall as a boosting marker did not occur in either group of texts. Will was the most frequent boosting modal verb in both corpora though it had a higher proportion in NNE texts (57.14\% vs. $51.89 \%)$. Must held the second position in both groups of research articles although it appeared with a higher proportion in NE texts (48.1\% vs. $42.85 \%$ ) (see Table 5).

\begin{tabular}{llllll}
\hline & \multicolumn{2}{c}{ NE texts } & & \multicolumn{2}{c}{ NNE texts } \\
\cline { 2 - 3 } \cline { 5 - 6 } & Total & Percentage & & Total & Percentage \\
\hline Will & 96 & $51.89 \%$ & & 8 & $57.14 \%$ \\
Must & 89 & $48.1 \%$ & & 6 & $42.85 \%$ \\
Shall & 0 & 0 & & 0 & 0 \\
Total & 185 & $100 \%$ & & 14 & $100 \%$ \\
\hline
\end{tabular}

Table 5: The frequency and distribution of the types of boosting modal verbs in NE and NNE texts

\section{Discussion}

As it was stated earlier, the current study aimed at investigating how and to what extent native and non-native writers in the linguistics research articles written in English took their stances toward propositions and readership employing modal auxiliaries via a contrastive analysis study.

The findings revealed that the NE authors tended to apply modal auxiliaries more than twice as many as those employed by the NNE authors. Using modal verbs more frequently by the NE writers is compatible with the results of Orta's (2010) study in which it was found that the NNE writers used fewer modal verbs than the NE writers. The difference between the English and Persian authors can be explained by two probable justifications. First, the use of modal auxiliaries might be confined by Persian cultural and/or linguistic conventions and properties. Second, the notions carried by modal auxiliaries could be expressed through some other modality resources in Farsi. Additionally, the two groups of authors were also different in the pattern of their use, that is, the proportion of modal auxiliaries did not follow an equal pattern. Unlike the English authors, the Persian writers used can approximately half of the times. Similarly, could as the tentative member of can occurred in the NNE texts more than the NE ones. 
May was the auxiliary applied by the two groups of writers with nearly the same proportions. In the rest of auxiliaries, the proportions were higher in the NE texts. The reason why the proportion of can was so high in the NNE texts in comparison with the NE ones might be the fact that, in Persian language, the equivalent of can (tavänestan) is used with a high proportion in both written and spoken forms. Here, transfer from the mother tongue can plausibly justify the high occurrences of can in the English texts by Iranian authors. This reason can be documented by another finding demonstrated in the pie charts. That is, the higher proportion of the tentative member of can (could) in the NNE texts. Another possible reason, for the presence of the wide proportion of can in the NNE texts may be the Iranian writers' lack of enough familiarity and knowledge about the meanings and usages of the remaining auxiliaries specifically would and might. This might have led them to compensate their lack of enough knowledge with the high use of can. On the other hand, the lower proportion of the rest of modal auxiliaries (might, must, will, would, and should) in the NNE texts may be the result of the avoidance strategy adopted by the Persian writers since some of these modals do not have any direct equivalents in Persian language. Therefore, they seem to be problematic areas to be learnt by Persian second language learners. Furthermore, they are less frequent in Farsi and this might have transferred to the Iranian authors' English writings. May possessed almost the same proportion in the two corpora. It seems that may was easier for the NNE authors to be comprehended and learned functionally than the other ones. Contrary to our expectation, the proportion of shall in the NE texts was lower than that in the NNE ones. The potential explanation for achieving such a result is the extremely low frequency of this modal in the two corpora. The low frequency of shall in academic texts was also confirmed by Biber et al. (1999). Therefore, the relatively higher proportion of shall in Persian corpus may be due to chance.

In the second phase of analysis, the results illustrated in the six bar graphs are an obvious indicator of the fact that both groups of writers had a noticeably high tendency towards using the modal verbs in their epistemic functions rather than their deontic ones except for will in the NNE texts. Interestingly, will was the only modal verb in the native corpus which was used in its deontic meaning far more than the other modal verbs in their deontic meanings. However, in the NE texts, the frequency of will in its deontic function did not exceed its epistemic one. The relatively high use of will in its deontic meaning by the two groups of writers implies that they both tended to inform the readers of the forthcoming organization of the text. The examples 13 and 14 from the corpus clarify the point.

(13) Before doing so, however, two specific languages from the sample will be examined in considerable detail (NE texts).

(14) We will examine and elaborate on the properties of LVs in section 3.1 and the semantic and morphosyntactic features of nominal PVs will be presented and focused on in section 3.2. (NNE texts).

The reason behind the NNE writers' higher use of will in its deontic meaning could also be attributed to its relatively straightforward deontic meaning in its volition function (future tense). The similarities observed in the general patterns of modal deployment may be due to the fact that both groups of the writers seem to be relatively aware and follow the generic conventions of research articles although both of them had been under the influence of their 
cultural backgrounds and linguistic properties. This has been manifested in the existing differences in the frequency of occurrences of modal verbs in the texts.

Furthermore, it can be concluded that both groups of the writers used modal verbs as devices of expressing stances toward their content and readership. Nevertheless, the NE writers' stances in the discourse appear to be much more than the NNE writers'. That is, stance-taking strategies via modal auxiliaries are dominant in the NE texts.

One probable explanation for such a finding can be lack of sufficient knowledge on the part of the NNE writers about the English language in general and modal auxiliaries and their uses in particular. As stated earlier (Chen 2012; Lorenzo 2008), modal auxiliaries can be an area of difficulty not only for non-native writers but also for native ones since they are polysemous and multi-functional. The condition becomes much worse when it comes to inexperienced non-native authors. The other possible reason is that, in foreign language education systems, the language is learned mostly via classroom instructions, and learners are rarely provided with or experience natural language learning situations to acquire pragmatic aspects of the language. Additionally, it appears that classroom instructions do not necessarily pay much attention to this crucial dimension of language to compensate for the lack of natural language learning situations. The third possible interpretation which can account for the Persian writers' reluctance to use auxiliaries as stance-taking markers is that they might express their stances toward the knowledge claims and addressees through some other linguistic devices (such as modal nouns, modal adjectives, etc.) which are more common in their language. This is a matter for future research.

In the ultimate phase of the study, modal auxiliaries were viewed from a different perspective. That is, they were assessed to see how the two groups of writers tended to express their stances concerning hedging and boosting strategies. As the results indicated, both groups of the writers had a high inclination toward hedging rather than boosting strategies. This finding is supported by some other previous studies (e. g. Hyland 1998; Kondowe 2014) working on hedging and boosting devices. The tendency to prefer more hedging devices by the two groups can be interpreted by the principles of academic communities especially in "soft" sciences such as linguistics (Sameri/Tavangar 2013; Vazquez/Ginger 2008) and the generic features of research articles (Hyland 1995). The percentage column in Table 3 indicated that the proportion of the hedging devices to the boosting markers in the NNE texts was higher than the proportion of the hedges to the boosters in the NE ones. Two different reasons can explain the finding. First, it seems that will and must as boosting devices are among the auxiliaries which EFL non-native writers have much problems in comprehending their epistemic meanings. Second, this can be associated with the existence of the auxiliary can among the hedging markers which had been used far more by the NNE writers in comparison with the NE ones, possibly as the result of transference (see pie charts and bar graphs). This has been previously discussed in the first part of the discussion.

The information in Table 3 also showed that the NE writers employed the hedging devices approximately twice as much as the NNE ones. Some possible reasons can justify this issue. First, culturally it appears that Persian non-native writers do not tend to hedge their propositions and are willing to speak and write categorically (Shokouhi/Talati-Baghsiahi 2009). Second, concerning the politeness strategies, the NNE writers may have some other linguistic 
devices at their disposal in the discourse to express their politeness and save the negative face of the audience. Furthermore, they might be unaware of pragmatic notions of these devices expressing politeness. Different cultures may have different outlooks at expressing politeness towards their addressees. The NE writers also used the auxiliary verbs as boosting resources in much higher frequency comparing with the NNE writers. This shows that the NNE authors demonstrated not much interest in applying both the hedging and boosting devices. That is, they were not willing to indicate their stances clearly in the texts. Consequently, they prevented to establish their commitment and/or detachment to the truth value of the propositions. This can be a good reason to claim that the Persian non-native linguistics research writers were not much aware of the importance of stance-taking in academic discourse. This would possibly damage their reputation if the truth of the statements is not verified by the readers and the future findings.

Table 4, demonstrating the proportion of the hedging devices within each group in rank order, highlighted that more than half of the detachment procedures via modal auxiliaries as hedges had been expressed by the verb can. And the other five modal auxiliaries as the hedging devices (may, might, would, should, could) had taken the same role less than half of the times totally. This is a verification of the dominance of the word tavänestan (the equivalent of can) in Persian language and its transference to English which has been discussed fully earlier.

Table 5 indicated that the proportions of the three modal auxiliaries of will, must, and shall had the same rank order in the two groups of texts. This can be adopted as a fair interpretation for a similar generic pattern observed by the two groups of writers unlike their differences in their frequencies.

All in all, the Persian writers, compared with the native ones, showed a discrepancy in expressing their stances towards both their statements and the readers though minor similarities were detected in their stance-taking strategies in the genre of research articles. This led the researchers to conclude that stance-taking can be affected linguistically, culturally, and epistemologically.

\section{Conclusions and Implications}

The current study was to investigate, via a contrastive analysis, the distribution and use of modal auxiliary verbs as stance-taking devices in NE and NNE linguistics research articles. The findings revealed both differences and similarities. However, the differences exceeded the similarities a much higher degree. It can be concluded that either the Persian NNE authors of linguistics research articles might be relatively not much acquainted with the academic community conventions concerning stance-taking strategies through discourse, or they appear not to tend to take stances towards their propositions and might be inclined to express their statements in a more direct manner. In the former situation, the obtained findings suggest some implications for English language education system contributors. The authors suggest EAP material developers to include modal auxiliaries with their different functions into the educational content as important linguistic interactive sources expressing writers' stances through the academic texts. EAP lecturers and teachers are also advised to make themselves equipped with the knowledge of modal auxiliaries both semantically and pragmatically and to try to make the students conscious of the importance of such resources in academic writing. 
Moreover, they are encouraged to apply the proper methodologies and techniques to teach them appropriately, for instance, via using academic task-based activities such as writing review papers with a focus on using modal verbs, or giving academic texts to students and asking them to identify modal verbs as well as their functions. In a similar vein, researchers are suggested to learn the appropriate use of modal auxiliaries in order to express themselves properly in their texts and in interacting with the readers. Finally, given that considerable differences were found between the native and non-native writers in the current study as well as Orta's (2010) study, linguistics researchers are advised to carry out similar comparative studies in other cultures to illuminate the issue.

Yet, the study is not claimed to be conducted without any limitations. The research is limited merely to linguistics discipline and a limited number of research papers. Further research is needed to be carried out on other fields of study. Other researchers are also recommended to conduct similar studies on larger corpora in order to make the findings more generalisable.

\section{References}

Ahmad, Ummul/Mehrjooseresht, Maryam (2012): "Stance adverbials in engineering thesis abstracts". Procedia - Social and Behavioral Sciences 66: 29-36. doi: 10.1016/j.sbs pro.2012.11.244.

Almeida, Francisco Alonso/Vazquez, Nila (2010): Modality and stance in the modern English part of the corpus of early English recipes. www.um.es/lacell/aelinco/contenido/pdf/80.pdf [15.04.2015].

Biber, Douglas et al. (1999): Longman grammar of spoken and written English. Harlow: Pearson Education.

Brown, Penelope/Levinson, Stephen C. (1987): Politeness: Some universals in language usage. Cambridge: Cambridge University Press.

Chen, Zhenzhen (2012): "Expression of epistemic stance in EFL Chinese university students' writing”. English Language Teaching 5/10: 173-179. doi: 10.5539/elt.v5n10p173.

Hinkel, Eli (1997): "Indirectness in L1 and L2 academic writing". Journal of Pragmatics 27: 361-386. doi: 10.1016/S0378-2166(96)00040-9.

Hinkel, Eli (2005): "Hedging, inflating, and persuading in L2 academic writing". Applied Language Learning 15/1: 29-53.

Hsieh, Chia-Ling (2009): "Epistemic stance taking in Chinese media discourse". Lilun Yuyanxue Yanjiu 3: 1-35.

$\mathrm{Hu}$, Guangwei/Cao, Feng (2011): "Hedging and boosting in abstracts of applied linguistics articles: A comparative study of English- and Chinese-Medium Journals". Journal of Pragmatics 43: 2795-2809. doi: 10.1016/j.pragma.2011.04.007.

Huschová, Petra (2015): "Exploring modal verbs conveying possibility in academic discourse". Discourse and Interaction 8/2: 35-47. doi: 10.5817/DI2015-2-35.

Hyland, Ken (1995): “The author in the text: Hedging scientific writing". Hong Kong Papers in Linguistics and Language Teaching 18: 33-42.

Hyland, Ken (1996): "Writing without conviction? Hedging in scientific research articles". Applied Linguistics 17/4: 433-454. doi: 10.1093/applin/17.4.433.

Hyland, Ken (1998a): "Boosting, hedging and the negotiation of academic knowledge". TEXT 18/3: 349-382. 

as Stance-Taking Devices in Linguistics Research Articles

Hyland, Ken (1998b): "Exploring corporate rhetoric: Metadiscourse in the CEO's setter". The Journal of Business Communication 35: 224-245.

Hyland, Ken (1999): "Disciplinary discourses: Writer stance in research articles". In: Candlin, Christopher/Hyland, Ken (eds.): Writing. Texts, processes and practices. London, Longman: 59-78.

Hyland, Ken (2000): "Hedges, Boosters and lexical invisibility: Noticing modifiers in academic texts". Language Awareness 9/4: 179-197. doi: 10.1080/09658410008667145.

Hyland, Ken (2006): "Medical discourse: Hedges". In: Brown, Keith (ed.): Encyclopedia of language and linguistics. $2^{\text {nd }}$ ed. Oxford, Elsevier: 694-697.

Hyland, Ken/Milton, John (1997): "Qualification and certainty in L1 and L2 students writing”. Journal of Second Language Writing 6/2: 183-205. doi: 10.1016/S1060-3743(97) 90033-3.

Hyland, Ken/Tse, Polly (2004): "Metadiscourse in academic writing: A reappraisal". Applied Linguistics 25/2: 156-157. doi: 10.1093/applin/25.2.156.

Kondowe, Wellman (2014): "Hedging and boosting as interactional metadiscourse in literature doctoral dissertation abstracts". International Journal of Language Learning and Applied Linguistics World 5/3: 214-221.

Lorenzo, Danica Salazar (2008): Modality in student argumentative writing: A corpus-based comparative study of American, Filipino and Spanish novice writers. Unpublished $\mathrm{PhD}$ Thesis, University of Barcelona.

Orta, Ignacio Vázquez (2010): “A contrastive analysis of the use of modal verbs in the expression of epistemic stance in Business Management research articles in English and Spain”. Iberica 19: 77-96.

Reilly, Judy/Zamora, Anita/McGivern, Robert F. (2005): “Acquiring perspective in English: The development of stance”. Journal of Pragmatics 37: 185-208.

Sameri, Motahareh/Tavangar, Manoochehr (2013): "Epistemic modality in academic discourse: A cross-linguistic and cross-disciplinary study". Iranian EFL Journal 9/4: 127147.

Shokouhi, Hossein/Amrollah Talati-Baghsiahi (2009): "Metadiscourse functions in English and Persian sociology articles: A study in contrastive rhetoric". Poznan studies in contemporary linguistics 54/4: 549-568.

Vázquez, Ignacio/Giner, Diana (2009): "Writing with conviction: The use of boosters in modelling persuasion in academic discourses". Revista Alicantina de Estudios Ingleses 22: 219-237. 